\title{
WAVELET BASED Fingerprint AUTHENTICATION SYSTEM: A REVIEW
}

\author{
Rakesh Verma \\ Department of Electronics and Communication Engineering, \\ NIT Kurukshetra \\ Kurukshetra, India
}

\begin{abstract}
Biometric recognition refers to the use of biological characteristics for identification and verification of individuals. Use of biometric is increasing nowadays because biometric characteristics are difficult to replicate and lifelong. Applications which require only the authorized persons to access the resources are information systems, National id systems, voter and driver registrations, documentations, military area and ATMs. Among all biometrics, fingerprints is the most widely used and accepted by the user as the acquisition of fingerprint image is minimally invasive and require little hardware. Traditional fingerprint identification algorithm based on Minutiae Extraction, Discrete cosine transform (DCT) and fast Fourier transform require so much computation. Wavelet based algorithm do not require any preprocessing and post processing steps and hence is the key for low cost fingerprint identification system. This paper presents the overview of different fingerprint recognition system. In comparison to older fingerprint recognition system that is based on minutiae and FFT, the wavelet based system has high recognition rates.
\end{abstract}

\section{KEYWORDS}

Biometrics, Fingerprint Recognition, Minutiae, FFT, Wavelet

\section{INTRODUCTION}

Personal identification has been a great research area with various solutions proposed. Applications that require accurate and reliable methods of personal identification are accessing buildings or facilities, secure electronic banking, credit cards, ATMs, mobile phones and secure access to military area. Personal Biometrics refers to identification of a person based on the physical and behavioral characteristics. Token-based and password based approaches are used for the existing security system, but these methods are not very secure. Tokens and access cards may be duplicated or stolen. Passwords and personal identification number numbers may also be stolen electronically. Biometric identifiers are more reliable than tokens or password based methods. Biometric characteristic do not change with time so they are advantageous over the traditional identification method.

Biometrics identifies a person based on biometric characteristics such as finger prints, hand geometry, iris, retina and face [1]. Biometric is used to achieve a "positive identification" with an error rate of $0.001 \%$ [2]. Finger prints biometric have one of the highest levels of reliability [3, 4].

DOI : 10.14810/elelij.2016.5105 
Forensic expert use fingerprint biometrics technique in criminal investigations [5]. Traditional fingerprint recognition system uses minutiae based approach [6].The minutiae based fingerprint recognition system always achieves very high accuracy. The shortcomings of the minutiae based fingerprint recognition system are:

(a) These systems are not useful for noisy or low resolution images. They always require high quality of images but storing of high quality images requires large amount of storage.

(b) These systems are slow for the real time applications as these systems requires steps like preprocessing, registration and orientation flow estimations which leads to slowness of system.

(c)These systems have low recognition rates as these not deal with translated, rotated and scaled images.

The alternative to minutiae based approach is the image based approach. In this approach we extract features directly from the original image without preprocessing so they are computationally efficient. Study shows that use of wavelet transforms on fingerprint texture increase the recognition rates [7].In a fingerprint texture is a pattern of ridges and valleys. The texture analysis is used for the analysis and classification of images [8]. The approaches that can be used in texture analysis are statistical, spectral and structural [9]. In most texture analysis schemes the images are analyzed at one single scale. Using wavelets multidirectional and multi resolution property local and directive information from the texture can be obtained which is utilized to increase the recognition rates. Some image based approach require elaborate algorithm to make them robust to low image quality. MR (Multi Resolution) techniques [10, 11], improves the classification accuracy of biological images. They are fast and efficient to compute and provide space-frequency localized information in sub bands.

The paper is organized as follows: In Section-II, existing work done in fingerprint recognition is studied. Section-III deals with the theory of Wavelet Transform and discrete wavelet transforms in brief. In Section-IV, different approaches for fingerprint recognition along with their limitations are presented followed by conclusion in section V.

\section{SHORT REVIEW ON FINGERPRINT RECOGNITION TECHNIQUES}

S.S.Gornale et al., [12] presented discrete wavelet transform (DWT) image de-nosing algorithm. Image denoising was done by time invariant properties of un-decimated discrete wavelet transforms. Wavelet transform are used to omit down-sampling in the forward transform and up sampling in the inverse transform. Zin Mar Win [13] proposed a fingerprint recognition system based on Myanmar National Registration Cards (NRCs). Gabor filters were used to enhance the low quality images. Correlation-based approach was used for the fingerprint matching which increases the recognition rates. Amjad Ali et al.,[14] introduced a GLCM based fingerprint recognition algorithm. Core point was found using Poincare Index method. Diffusion Coherence technique was used to enhance the dominant fingerprint region around the core point. Seven significant statistical descriptors have been calculated using the GLCM with different pixels separation distances between pixels in four orientations. K-Nearest Neighbor (KNN) was used as similarity measure. Rakesh Verma et al.,[15] proposed a wavelet based texture pattern recognition method for fingerprint recognition. The approach used gives high recognition rates in comparison to older fingerprint recognition system based on Fast Fourier Transform (FFT) and Minutiae Extraction. Zin Mar Win et al.,[16] presented a Discrete Wavelet Transform based 
approach for fingerprint recognition. Region of interest (ROI) was extracted around the core point of the finger.

Gabor filters were applied to cropped images for enhancement. Standard deviation and Energy were used as texture feature. Euclidean distance metric was used as similarity measure. Mesbah Uddin Khan et al. [17] proposed a fingerprint recognition system based on Singular Points. Singular points (cores and deltas) were used for fingerprint matching. Orientation estimation with the use of directional fields detected the maximum number of singular points (cores and deltas) in a fingerprint and increases the recognition rates. Yasir Ali Shah et al., [18] presented a Discrete Cosine Transform and Discrete Wavelet Transform based fingerprint authentication system. Discrete cosine transform (DCT) were used to transform the images into frequency components. DWT was used to decompose frequency components into lower, medium and higher frequency sub bands. Feature vector was extracted from the decomposed frequency bands using Linear Discriminant Analysis (LDA). Ting Tang [19] presented an image-based fingerprint recognition method using wavelet transformation. Reference point and rotation alignment algorithms were used to find region of interest (ROI). Wavelet transform were applied on ROI to find local and global features (Mean energy, Standard deviation and entropy). Euclidian distance was used for fingerprint matching. Liang -Wei Chen et al., [20] proposed a fingerprint recognition system based on Invariant moment features. Region of interest (ROI) was extracted and segmented into several rings. Hu invariant moment features were calculated and Euclidian distance was used for fingerprint matching. Maddu Kamaraju et al., [21] introduced Digital Signal Processor (DSP) based embedded fingerprint recognition system. Fingerprint images were enhanced using Gabor filter. Minutiae features were extracted from the enhanced images which increases the recognition rates. Nushrat Humaira et al.,[22] presented Curvelet feature based Fingerprint recognition system using Fourier enhancement. Fingerprint image was aligned using 'Poincare Index value' method. Feature vector set from image was extracted using Curvelet transformation in different scale and orientation. Classifier K nearest neighbor was used to classify Curvelet co-efficient matrix for reorganization of the specific fingerprint. Vaishali Pawar and Mukesh Zaveri [23] implemented a graph clustering and matching algorithms based fingerprint recognition system. Structural features of the minutiae from the fingerprint were extracted. Attributed graph from the fingerprint was taken to relate the entire feature with each other. For matching Euclidean distance based K-NN clustering technique was applied to feature set. The proposed approach reduces the time to one third in comparison without clustering. K.Usha and M.Ezhilarasan [24] proposed a Haar-Wavelet Transform based finger knuckle print (FKP) recognition technique. Knuckle image was transformed into a subset of its feature space known as 'Eigen Knuckle' using Haar Wavelet. Eigen vectors were formed by the principle components and local space variations. Knuckle images for personal identification were matched by means of a classifier using correlation. Ali Akbar Nasiri and Mahmood Fathy [25] proposed an algorithm based on flow curve and clustering for the detection of fingerprint reference point. The key feature of the proposed approach is that it was robust to noise. Masao Yamazaki et al. [26] presented a scale invariant feature transform (SIFT) approach for fingerprint authentication for smart phones. In proposed approach two different images for the same finger were merged to replace the conventional minutiae method. The proposed approach is used with touch sensor on smart phone. Satishkumar Chavan et al. [27] presented a fingerprint authentication system based on filter bank. Gabor filter bank were used to extract the fingerprint feature. Euclidian distance was used for similarity score of feature vectors. Satish S Bhairannawar et al. [28] proposed a fingerprint recognition system based on O2D-DWT architecture and modified CORDIC-FFT. 
O2D-DWT architecture was used to generate four sub bands. From LL sub band feature vector was generated using the optimized Fast Fourier Transform (OFFT) and CORDIC processor. Euclidean Distance was used for fingerprint matching.

\section{WAVELET TRANSFORM AND DISCRETE WAVELET TRANSFORM (DWT)}

\subsection{Wavelet Transform}

Wavelet transform is a powerful tool to analyze different signals. A wavelet is a waveform of effectively limited duration that has an average value of zero [29]. Wavelets are used to break the data into different frequency components and these frequency components can be studied with a resolution matched to its scale. So wavelet transform provides the time and frequency information simultaneously. The advantage of wavelet transform over traditional Fourier methods is they can analyze signals with discontinuities and sharp spikes. Few commonly used wavelets families are named as Haar, Symlets, Daubechies, Coiflets, Shannon.Fig.1 shows some single wavelet families. The choice of wavelet family depends on application requirement. A user can choose wavelets with simple mathematical expressions, compactly supported wavelets, wavelets with simple associated filters and smooth wavelets etc.
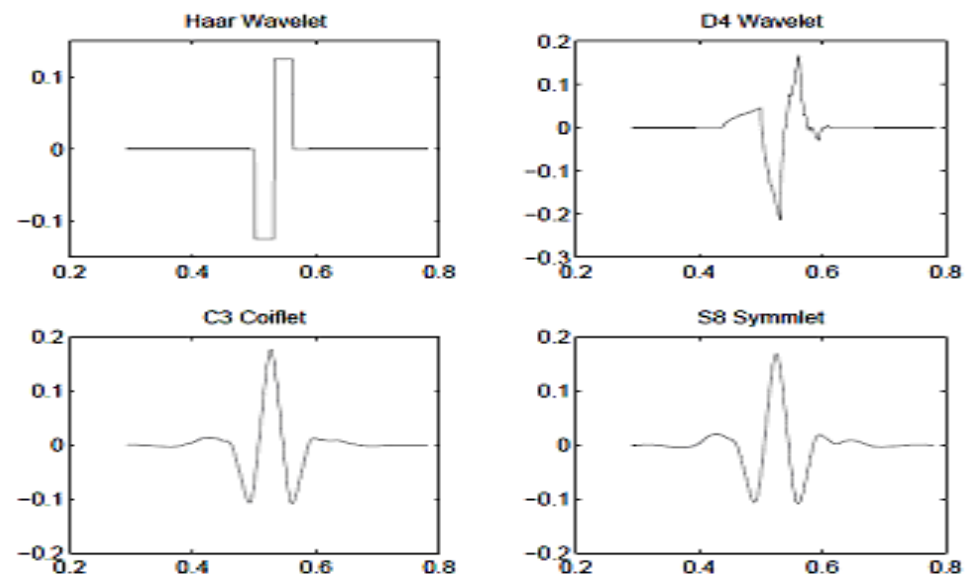

Figure1. Some single wavelet families [29]

Wavelets are used for a number of applications, some examples are: Speech recognition, Texture analysis \& classification, Fingerprint verification, Classification \& clustering, Data compression, Document analysis, heart-rate and ECG analyses. A signal is divided into a bunch of signal by the Wavelet Transform. The bunched signals represent the same signal, but all corresponding to different frequency bands. The principle advantage is they provide time and frequency information simultaneously i.e at what time interval what frequency bands exist. The Wavelet transform of a function $\mathrm{f}$ is given by

$W f(a, b)=\frac{1}{\sqrt{a}} \int_{-\infty}^{\infty} f(t) \psi(t-b / 2) d t$ 
Where $\mathrm{a} \& \mathrm{~b}$ are frequency and time respectively and $\psi(\mathrm{t})$ is known as mother wavelet.

\subsection{Discrete Wavelet Transform}

Discrete Wavelet Transform (DWT) is used to provide the time frequency representation. DWT is used to divide the image into four sub bands as shown in Fig. 2 (a). These sub bands represent octave-band decomposition and are logarithmically spaced in frequency [30]. The sub bands labeled with LH1 and HL1 gives horizontal detail and vertical detail of the image respectively. The sub bands HH1 and LL1 represent the detail images (High frequency) and approximation image (Low frequency).Approximation image is same as the original image. Approximation image LL1 is further decomposed to get the second level wavelet coefficients as shown in Fig. 2 (b). Approximation image LL2 is further used to get next level wavelet coefficients. This process is used to obtain final scale. The main advantage of DWT is multi-scale representation of image. By using the wavelets, given image can be analyzed at various levels of resolution.

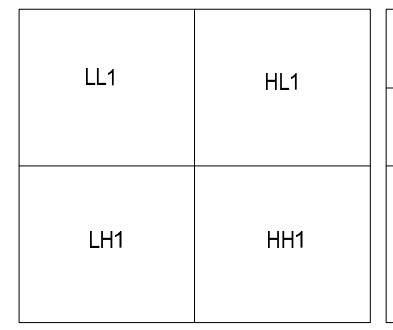

(a) One level

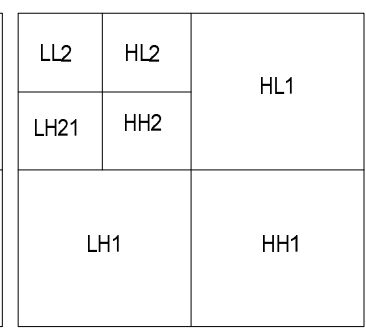

(b) Two level

Figure2. Image Decomposition

\section{FINGERPRINT RECOGNITION APPROACHES}

In this section various approaches to fingerprint recognition are studied in detail.

\subsection{The Henry System approach}

Fingerprint database may consist of millions of fingerprints. It leads to long response time and hence not used for real time applications. Fingerprint classification is used to reduce the number of comparison so reduces the response time. Henry [31] presented a fingerprint classification approach that classifies fingerprint records according to gross physiological characteristics and hence reduces the response time to search large numbers of fingerprint records. This system is used to assign a working formula to a set of fingerprints. Here numerical values are assigned to each finger starting from right thumb as number 1 and the left pinky as end with number 10 . The

Henry system also assigns a numerical value to fingerprint whorl patterns. Fingers 1 and 2 are assigned a value 16, fingers 3 and 4 are assigned a value 8 and so on. Final two fingers have a value of 1 . Fingers which only have an arch or loop pattern have a value of zero. These values are used to form a numerical description of fingerprints. Henry system used 10 fingerprint patterns from both hands for the classification of an individual. Images of various fingerprint patterns are illustrated below: 


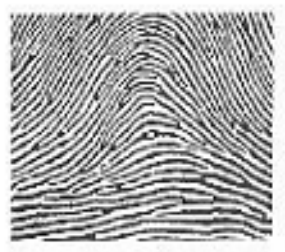

Arch

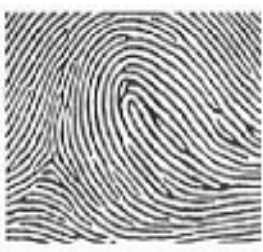

Loop

Figure3. Fingerprint Patterns in Henry System

The shortcoming of Henry classification systems are: (a) It uses non uniform classification proportion. (b) Some fingerprint images may be classified into different Henry classes.

\subsection{Minutiae Based Approach}

A fingerprint is the pattern of ridges and valleys on the finger [32]. Each person has different patterns of ridges and valleys with the permanent uniqueness. Research on fingerprint recognition showed that fingerprints are not only distinguished by pattern of ridges and valleys but also distinguished by some abnormal points on the ridges and valleys which are known as Minutia. The most significant minutia points are termination and bifurcation which are shown Fig. 4. Immediate ending of a ridge is known as termination and the point from which two branches are derived is known as bifurcation.

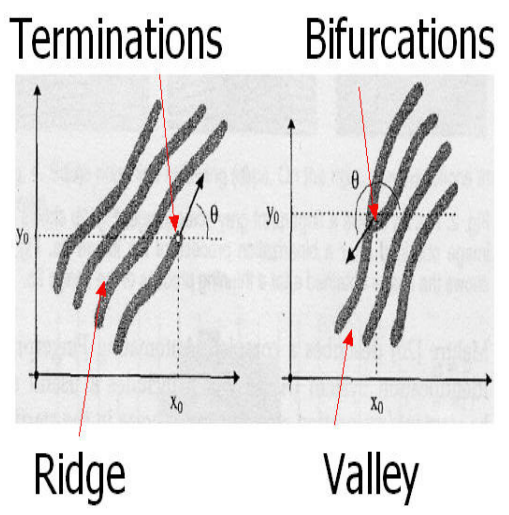

Figure 4. Minutia (Termination and Bifurcation)

The minutiae set can be represented as a point pattern and this reduces the fingerprint verification problem to a minutiae point pattern matching problem. One of the popular methods for the fingerprint recognition is Minutia-based extraction. Landmarks such as termination, ridge bifurcations and ridge ends are extracted with a reliable minutiae extraction algorithm. The minutia extraction algorithm mainly consists of three components, orientation field estimation, ridge extraction, and minutiae extraction and post processing. Different steps for minutia extraction algorithm in illustrated in Fig. 5. 


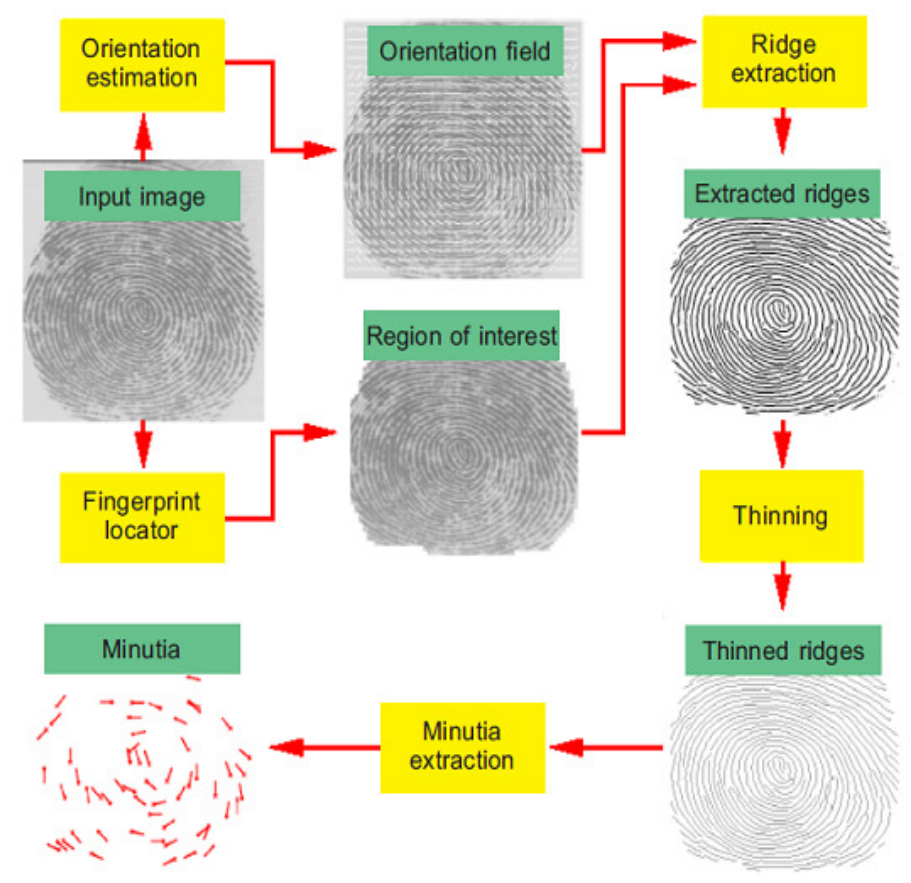

Figure 5. Flowchart of Minutiae Extraction Algorithm [33]

A fingerprint recognition system that uses minutiae extraction algorithm always achieves very high accuracy. The shortcomings of this approach are:

(a) These systems require high quality fingerprint images.

(b) These systems are slow for real time applications.

(c) These systems have low recognition rates.

\subsection{Image Based Methods}

Image-based approaches do not require the extensive pre-processing. It means the whole image as an input to extract the features. WAN AZIZUN WAN ADNAN et al., in 2004 proposed a simple technique for fingerprint recognition which matches the fingerprint images based on the features extracted in the wavelet transform domain. The Image based algorithm is shown in Fig. 6.

Digitized image of fingerprint is preprocessed to obtain the binary image. The two dimensional Discrete Wavelet Transform is applied on the binarized image and after that one dimensional DWT is applied. Finally, a wavelet-based $4 \times 1$ feature row vector is obtained. This feature vector has information about the slopes of image. Identification can be made by comparing this feature vector with other fingerprint features. 
Electrical and Electronics Engineering: An International Journal (ELELIJ) Vol 5, No 1, February 2016

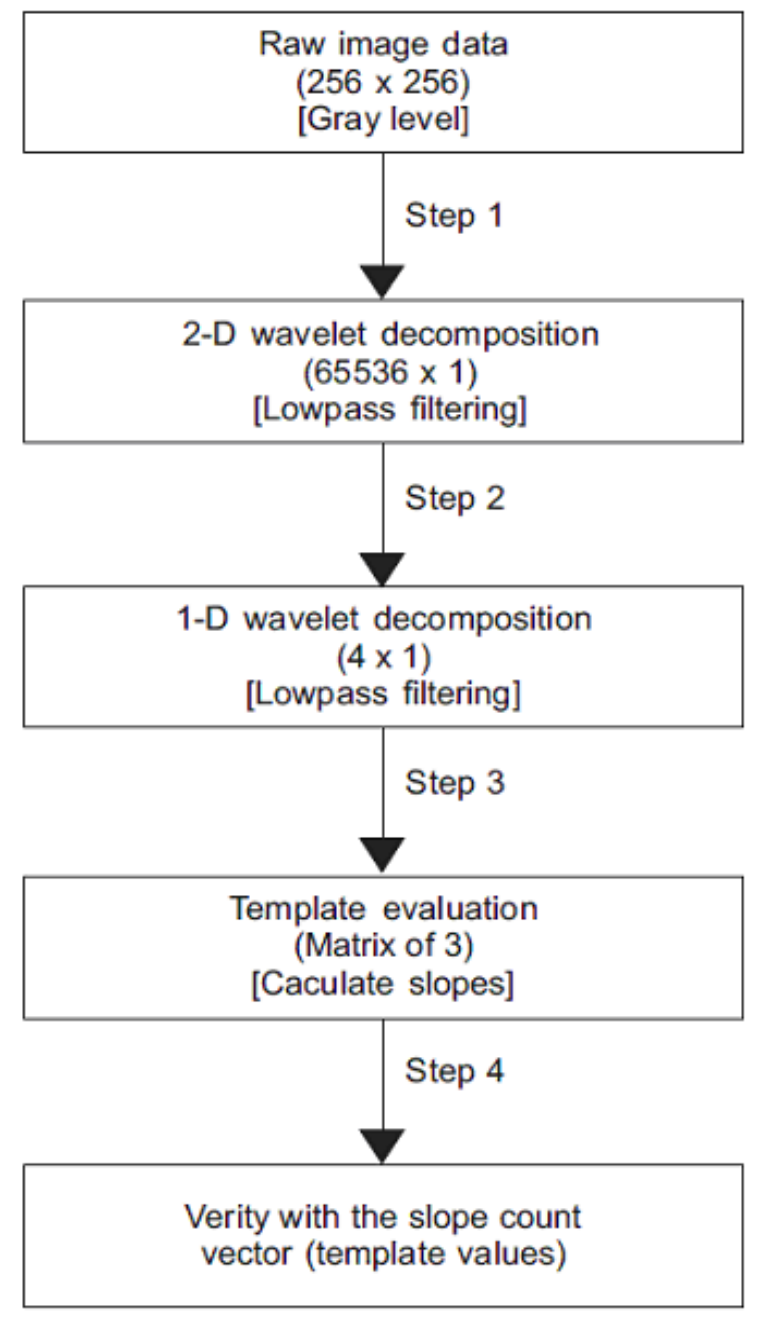

Figure 6. Block diagram of Fingerprint Recognition Algorithm using DWT

\subsection{The Pattern Recognition Approach}

The pattern recognition system is divided into five subsystems. First step is the Data generation. It converts the 3-dimensional print of the image into a usable gray-scale image. The second subsystem is used to preprocess the gray scale image. Feature extraction follows the pre-

processing. A unique feature vector is generated within this subsystem for the data that was generated in the first step. Feature extraction is followed by classification. The classifier used in this subsystem was trained on the vectors generated during the feature extraction phase. Classification is used to find the identity of the fingerprint. The results of the classifier are evaluated in the post processing stage. The functional block diagram of pattern recognition approach is shown in Fig.7. 


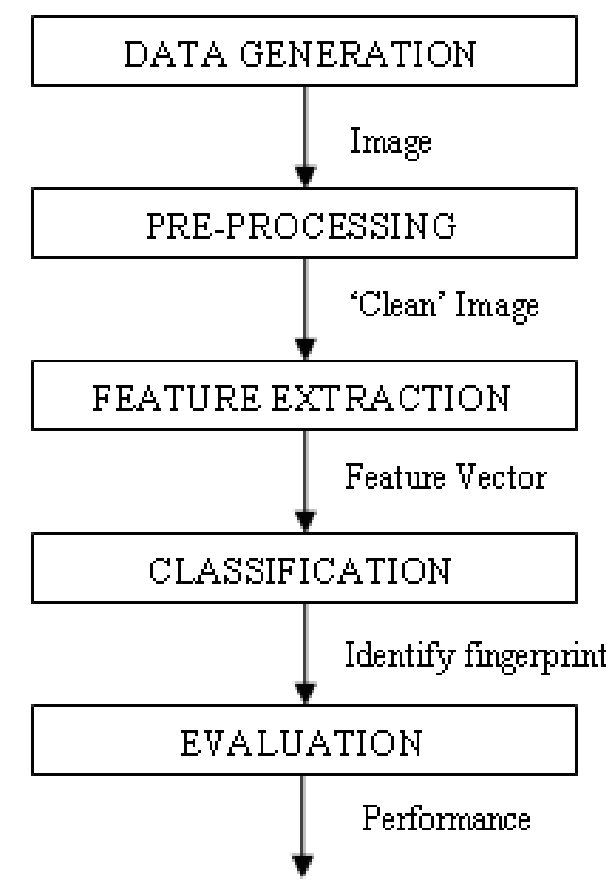

Figure7. Functional block diagram of pattern recognition approach [13]

Texture feature of the images are used in the pattern recognition approaches for classification. The disadvantage of this approach is that it analyzes the image at one single scale.

\subsection{Wavelets and pattern recognition approach}

Image recognition using pattern matching techniques gives less information about the texture as images are analyzed at single scales. The use of wavelet in pattern recognition approach improves the classification accuracy as the images can be analyzed by multi scale representation of texture [34]. Wavelets direction resolving property can be used to extract information in three directions (horizontal, vertical and diagonal) from texture which increases the recognition rates. So by the use of multi-resolution property of the wavelets more information of the texture can be obtained.

For fingerprint recognition, the algorithm follows steps:

Step1: Raw image is pre-processed to obtain binarized data form.

Step2: 2D-DWT is performed on binarized image to produce 1D co-efficient plot (large scale, high frequency)

Step3: 1D-DWT performed in step1 for a discrete representation at different scale Step4: stores particular fingerprint image in matrix form in database for later comparison Step5: tolerance within slope set the threshold of verification for matching input template 


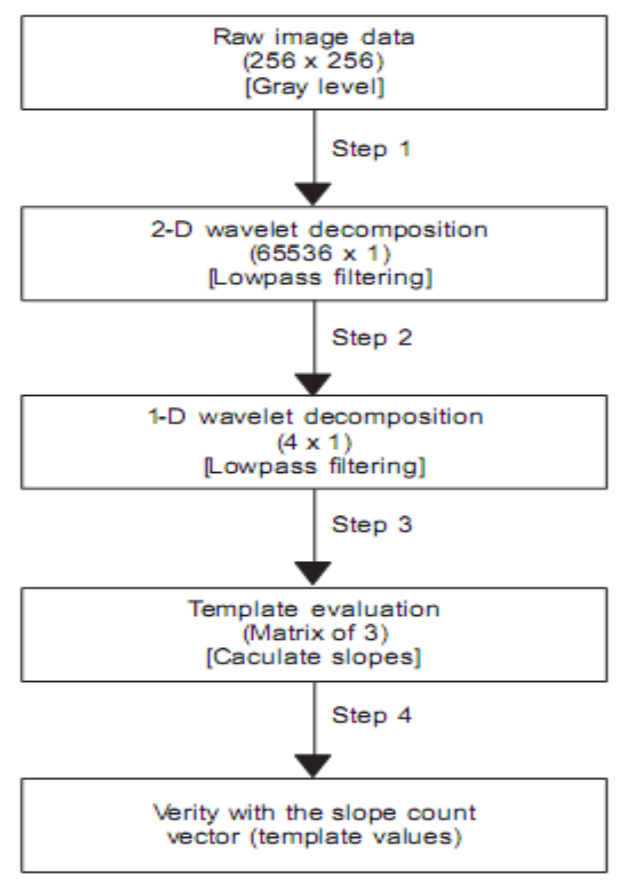

Figure8. Block diagram of fingerprint recognition algorithm

This algorithm is robust as DWT is rotation invariant transform.

\section{CONCLUSION}

This paper presented the different fingerprint recognition approaches along with the algorithms. The advantages and shortcomings of each approach are studied in detail. The traditional fingerprint recognition system takes more time for recognition because of preprocessing and post processing steps of images and hence become impractical .The wavelet transformed based system reduces the cost and response time of fingerprint systems as they eliminates any preprocessing and post processing of images. So Wavelet Transform has a vital role to increase the recognition accuracy of any fingerprint recognition system. The performance of the fingerprint recognition system increases due to multi-scale representation of fingerprint image by the wavelets. Recognition rates can be increased by the use of directional resolving power of wavelet transform. This local texture information increases the recognition rate. The use of multiresolution, compactness and denoising property of wavelets makes it is useful in fingerprint recognition system. 


\section{REFERENCES}

[1] A A.K. Jain, R. Bolle and S. Pankanti, Biometrics: Personal Identification in a Networked Society, Kluwer Academic Publishers, 1999.

[2] D. Polemi, "Biometric Techniques: Review and Evaluation of Biometric Techniques for Identification and Authentication, Including an Appraisal of the Areas Where They are Most Applicable," Final Report, April 1997.

[3] J.Berry and D.A.Stoney, " The history and development of finger printing in advances in fingerprint technology”, CRC Press, Florida, 2nd Edition, pp. 1-40, 2001.

[4] Emma Newham, "The Biometric report", SJB services, 1995.

[5] Federal Bureau of Investigation, "The Science of Fingerprints: Classification and Uses", US Government Printing office, Washington D.C. 1984.

[6] Maltoni, D. Maio, A.K. Jain, S. Prabhakar, "Handbook of Fingerprint Recognition", Springer, New York, 2003.

[7] W. Yongxu, A. Xinyu, D. Yuanfeng and Y. Li, "A Fingerprint Recognition Algorithm Based on Principal Component Analysis," Proceedings of IEEE Region 10 Conference TENCON, pp.1-4,1417, 2006.

[8] ASME B46.1, Surface texture (Surface roughness, waviness and lay), 1995.

[9] Yuanyan Tang "Status of Pattern Recognition with Wavelet Analysis" Front. Comput. Sci. China, pp. 268-294, 2008.

[10] T.Merryman, K. Williams, G. Srinivasa, A. Chebira, and J. Kovacevic, "A multiresolution enhancement to generic classifiers of subcellular protein location images," Proc. in IEEE Int. Symp. Biomed. Imaging, Arlington, VA, pp. 570-573, 2006.

[11] A. Chebira, T. Merryman, G. Srinivasa, Y. Barbotin, C. Jackson,R. F. Murphy, and J. Kovacevic, "A multiresolution approachto automated classification of subcellular protein location images", BMC Bioinformatics, 2007.

[12] S.S.Gornale, V.Humbe, R.Manza and K.V. Kale, "Fingerprint Image De-noising using MultiResolution Analysis (MRA) through Stationary Wavelet Transform (SWT) method," International Journal of Knowledge Engineering, vol. 1, no. 1, pp. 5-14, 2010.

[13] Zin Mar Win, Myint Myint Sein, "Fingerprint Recognition System for Low Quality Images", SICE Annual Conference Waseda University, Tokyo, Japan, pp. 1133-1137, 2011.

[14] Amjad Ali, Xiaojun Jing, Nasir Saleem, “GLCM-Based Fingerprint Recognition Algorithm”, IEEE Proceedings of IC-BNMT, pp 207-211,2011.

[15] Rakesh Verma,Anuj Goel, “Wavelet Application in fingerprint Recognition,” International Journal of Soft Computing and Engineering (IJSCE), Volume-1, no. 4, pp. 129-134, 2011.

[16] Zin Mar Win, Myint Myint Sein, "Texture Feature based Fingerprint Recognition for Low Quality Images”,IEEE , pp 333-338, 2011.

[17] Mesbah Uddin Khan,Muhammad Sheikh Sadi, "An Efficient Approach to Extract Singular points for Fingerprint Recognition", 7th International Conference on Electrical and Computer Engineering, Dhaka, Bangladesh, pp 13-16, 20-22 December 2012.

[18] Yasir Ali Shah, Nasir Ahmad, Muhammad Naeem, "Identification of Critical Bands in DCT Domain Representation for Fingerprint Recognition", International Conference on Automation \& Computing, Loughborough University, Leicestershire, UK, 2012.

[19] Ting Tang,Fingerprint Recognition Using Wavelet Domain Features", 8th IEEE International Conference on Natural Computation, pp. 531-534, 2012.

[20] Liang -Wei Chen, Chao- Rong Li, "Invariant moment features for fingerprint recognition", IEEE, pp 91-94, 2013.

[21] Maddu Kamaraju, Penta Ani! Kumar,” DSP based Embedded Fingerprint Recognition System”, 13th International Conference on Hybrid Intelligent Systems, pp. 6-11, 2013.

[22] Nushrat Humaira, Naila Bushra, Zannatul Firdous, Maruf Morshed Khan, Md.Monirul Islam, “Curvelet Feature Based Fingerprint Recognition Using Fourier Enhancement”, IEEE, 2013.

[23] Vaishali Pawar, Mukesh Zaveri, "Graph Based K-Nearest Neighbor Minutiae Clustering for Fingerprint Recognition”, 10th IEEE International Conference on Natural Computation, pp 675-680 , 2014. 
[24] K.Usha, M.Ezhilarasan, "Haar-Wavelet Transform based Finger Knukle Print Recognition", International Conference on Recent Trends in Information Technology, 2014.

[25] Ali Akbar Nasiri, Mahmood Fathy, "An effective algorithm for fingerprint reference point detection based on filed flow curves" International symposium on artificial intelligence and signal processing pp. 79-83, 2015.

[26] Masao Yamazaki, Dongju Li, Tsuyoshi Isshiki and Hiroaki Kunieda, "SIFT-based Algorithm for Fingerprint authentication on smartphone" 6th International Conference of Information and Communication Technology for Embedded Systems (IC-ICTES), 2015.

[27] Satishkumar Chavan, Parth Mundada, Devendra Pal,“ Fingerprint Authentication using Gabor Filter based Matching Algorithm” International Conference on Technologies for Sustainable Development (ICTSD-2015), 2015.

[28] Satish S Bhairannawar, Sayantam Sarkar, Raja K B, Venugopal K R, "An Efficient VLSI Architecture for Fingerprint Recognition using O2D-DWT Architecture and Modified CORDICFFT", 2015.

[29] Y.Y.Tan "Wavelet Theory and its Application to Pattern Recognition", World scientific, 2000.

[30] Marc Antonini, Michel Barlaud, Pierre Mathieu and Daubechies, "Image Coding using Wavelet Transform”, IEEE Transaction on Image Processing, Vol. 2, No. 2, pp. 205-220,1992.

[31] Wan Azizun Wan Adnan, Lim Tze Siang, \& Salasiah Hitam, "Fingerprint Recognition in Wavelet Domain," Jurnal teknologi, 41(D) Dis., pp 25-42, 2004.

[32] P. Komarinski, P. T. Higgins, and K. M. Higgins, K. Fox Lisa, "Automated Fingerprint Identification Systems (AFIS)”, Elsevier, Academic Press, pp. 1-118, 2005.

[33] A. K. Jain, S. Prabhakar and S. Chen, "Combining Multiple Matchers for a High Security Fingerprint Verification System. Pattern Recognition”, Letters. 20(11-13): pp. 1371-1379, 1999.

[34] K.Thaiyalnayaki, P. Varsha Parmar, S. Syed Abdul Karim "Finger Print Recognition using Discrete WaveletTransform" International Journal of Computer Applications, Volume 1, No. 24, pp.82-85, 2010. 